\title{
Positron binding in chloroethenes: Modeling positron-electron correlation-polarization potentials for molecular calculations
}

\author{
Haruya Suzuki, Takuma Otomo, Ryusei Iida, Yutaro Sugiura, and Toshiyuki Takayanagi॰* \\ Department of Chemistry, Saitama University, Shimo-Okubo 255, Sakura-ku, Saitama, Saitama 338-8570, Japan \\ Masanori Tachikawa \\ Graduate School of Nanobioscience, Yokohama City University, 22-2 Seto, Kanazawaku, Yokohama, Kanagawa 236-0027, Japan
}

(Received 22 September 2020; accepted 6 November 2020; published 25 November 2020)

\begin{abstract}
Positron binding energies and annihilation spectra are calculated using the positron-electron correlationpolarization potential model for a series of six chloroethene molecules. From detailed analysis of the positron densities and positron-molecule interactions, it was found that the positron is attracted by both the potential well around the negative end of the dipole moment and the $\pi$ electrons of the $\mathrm{C}=\mathrm{C}$ double bond. The positron annihilation spectra were simulated using the Feshbach resonance widths, which can be calculated from the couplings between the positron motion and vibrational motion. The importance of $\pi$ electrons in positron binding is discussed in terms of the calculated resonance widths.
\end{abstract}

DOI: 10.1103/PhysRevA.102.052830

\section{INTRODUCTION}

Over the past 20 years, numerous polyatomic molecules have been experimentally shown to have remarkable positron binding energies by using the low-energy positron beam technique [1-13]. It has now been established that the magnitude of the measured positron binding energy is generally correlated with various molecular properties, including molecular size, dipole moment, polarizability, and number of $\pi$ bonds in the molecule [8]; however, the atomic-level mechanisms of positron binding in molecules are not completely understood. Therefore it is crucial to develop reliable theories that can accurately predict positron binding energies for various polyatomic molecules with different molecular properties.

The development of quantum mechanical theory that can accurately describe positron binding in atoms and molecules has a long history [1]. It is well known that positron-electron correlation effects play an essential role, and sophisticated electronic-positronic structure theories, including the configuration interaction method [14-16], explicitly correlated Gaussian function method [17-19], and quantum Monte Carlo method [20-23], have previously been developed. In principle, these methods can provide accurate positron binding energies for small systems, although they cannot be applied to large polyatomic molecules containing many electrons owing to the prohibitively high computational cost.

Very recently, our group found that the practical correlation-polarization potential (CPP) model, which was

\footnotetext{
*Corresponding author: tako@mail.saitama-u.ac.jp

Published by the American Physical Society under the terms of the Creative Commons Attribution 4.0 International license. Further distribution of this work must maintain attribution to the author(s) and the published article's title, journal citation, and DOI.
}

originally employed in extensive positron-molecule scattering studies by Gianturco and co-workers [24-37], is very useful for describing positron binding in polyatomic molecules [38-40]. In this method, the short-range potential is approximated by a local positron-electron potential derived from the local electron density based on the density functional theory (DFT) framework, whereas the long-range attractive potential is described using dipole polarizabilities. We also found that the calculated positron binding energies can be improved by introducing the generalized gradient approximation [41,42] into the DFT-based potential. Another important advantage of the CPP model is that one can easily obtain the positron-molecule interaction potential as a function of nuclear coordinates. This enables calculation of the coupling between the positron and nuclear motions, which determines the resonance width for positron capture by a molecule. This is an important point because measurement of the positron binding energy of the molecule is carried out through observation of the vibrational Feshbach resonances in the annihilation spectrum as a function of positron collision energy. Thus the incoming positron is transiently captured into the vibrationally excited state of the target molecule at the resonance energy. We recently employed the CPP model to calculate the resonance-dominated positron annihilation spectra of carbon disulfide and benzene, where good agreement between theory and experiment was obtained [40].

In this work we calculated the positron binding energies and positron annihilation spectra of a series of six chloroethenes from monochloroethene (vinyl chloride) to tetrachloroethene using the CPP model. Experimental data are available for five of these chloroethenes, revealing that the positron binding energy is dependent on the number of chlorine atoms, which are expected to be negatively charged and preferentially attract the positron $[13,43,44]$. Another interesting finding of the experimental studies is that the measured positron binding energies of dichloroethenes are dependent on 
the chlorine substitution site; the positron binding energy of cis-1,2-dichloroethene was found to be greater than those of trans-1,2-dichloroethene and 1,1-dichloroethene. Moreover, it is worth mentioning that chloroethenes contain a $\pi$ bond, which may play a role in positron binding. We believe that the theoretical analysis of this series of chloroethenes may provide important insights for obtaining a detailed understanding of positron binding mechanisms as well as the role of $\pi$ bonds.

\section{THEORY}

The Hamiltonian of the system in which a single positron interacts with a molecule with fixed nuclei can be written as (in atomic units)

$$
\mathcal{H}(r, Q)=-\frac{1}{2 \mu}\left(\frac{\partial^{2}}{\partial x^{2}}+\frac{\partial^{2}}{\partial y^{2}}+\frac{\partial^{2}}{\partial z^{2}}\right)+V(r ; Q),
$$

where we use Cartesian coordinates $(x, y, z)$ to describe the positron position vector $r$. The reduced mass $\mu$ is taken to be the positron mass, meaning that we do not consider positronium formation at all in this work. Here, $Q$ collectively denotes the nuclear coordinates of the target molecule. The positronmolecule interaction potential $V$ can be approximated as

$$
V(r ; Q)=\sum_{i} \frac{Z_{i}}{\left|r-R_{i}(Q)\right|}-\int \frac{\rho\left(r_{\mathrm{e}} ; Q\right)}{\left|r-r_{\mathrm{e}}\right|} d r_{\mathrm{e}}+V_{\mathrm{cp}}(r ; Q),
$$

where $r_{\mathrm{e}}$ and $R_{i}$ are the collective position vectors of electrons of the target molecule and the position vector of the $i$ th positive nucleus (with $Z_{i}$ nuclear charge), respectively. The first term of Eq. (2) represents the positron-nucleus repulsive Coulomb interaction, and the second term represents the attractive interaction between the positron and electrons in the molecule with electron density $\rho$. The third term $\left(V_{\mathrm{cp}}\right)$ represents the correlation-polarization potential and can be approximated as [24-37]

$$
V_{\mathrm{cp}}(r ; Q)=\max \left[V_{\mathrm{corr}}(r ; Q), V_{\mathrm{pol}}(r ; Q)\right],
$$

where $V_{\text {corr }}$ and $V_{\text {pol }}$ are the short-range electron-positron correlation potential and long-range polarization potential, respectively. The correlation potential $V_{\text {corr }}$ can be written using the density functional expression including the generalized gradient approximation as $[41,42]$

$$
V_{\text {corr }}=V_{\text {corr }}^{\mathrm{LDA}} \exp \left[-\beta|\nabla \rho|^{2} / 3\left(\rho q_{\mathrm{TF}}\right)^{2}\right],
$$

where $V_{\text {corr }}^{\text {LDA }}$ is the local-density functional expression of Boroński and Nieminen [45], which was obtained from fitting to the correlation energy of one positron in a homogenous electron gas, $\rho$ is the electron density, and $q_{\mathrm{TF}}$ is the local Thomas-Fermi screening length. Notice that Eq. (4) with $\beta=0$ corresponds to the correlation potential with the local-density approximation (LDA). Previous studies have shown that the LDA approach frequently overestimates the positron-electron correlation energy [41,42], indicating that the nonlocal effect of electronic wave functions is playing a role in positron binding. In this work, the gradient parameter $\beta$ was taken to be an adjustable parameter. It may be worth mentioning that this value was set to 0.22 and 0.05 in the previous theoretical studies of Refs. [41,42], respectively. The long-range polarization potential in Eq. (3) can be approximated as

$$
V_{\mathrm{pol}}(r)=-\frac{1}{2 r^{6}} \sum_{i, j} x_{i} x_{j} \alpha_{i j}
$$

where $\alpha_{i j}$ is a Cartesian element $\left(x_{i}, x_{j} \in\{x, y, z\}\right)$ of the polarizability tensor.

The bound-state solution of the Schrödinger equation, the Hamiltonian of which is given by Eq. (1), provides the positron binding energy and its wave function $\psi_{i}$. We can obtain the positron scattering wave function $\psi_{f}$ at a specific collision energy from the same Schrödinger equation. The positron capture width $\Gamma_{v}^{e}$ can be calculated using these wave functions through Fermi's golden rule approximation as [40]

$$
\Gamma_{v}^{e}=2 \pi\left|\left\langle\psi_{f}\left(E_{v}, r ; Q\right)\left|V_{\mathrm{c}}(r ; Q)\right| \psi_{i}(r ; Q)\right\rangle\right|^{2},
$$

where the resonance energy is taken to be a specific vibrational energy level of the target molecule $\left(E_{v}\right.$, with $v$ denoting the vibrational quantum number). The interaction potential $V_{\mathrm{c}}$ can be approximated by the first-order Taylor series around the equilibrium nuclear coordinate, and then the integral can be easily calculated using the time-dependent golden rule approach [46-49]. It is worth mentioning that we employed the normal modes to describe the molecular structure, and thus the resonance width was calculated for each normal mode coordinate. The positron annihilation spectrum $\left(Z_{\text {eff }}\right)$ can be described using the well-known Breit-Wigner form [50,51],

$$
Z_{\mathrm{eff}}(E)=\frac{\pi}{k} \rho_{\mathrm{ep}} \sum_{v} \frac{g_{v} \Gamma_{v}^{e}}{\left(E-E_{v}\right)^{2}+\Gamma_{v}^{2} / 4},
$$

where $\Gamma_{v}$ and $g_{v}$ are the total width and degeneracy of the vibrational state, respectively. In Eq. (7), $E, k$, and $\rho_{\mathrm{ep}}$ are the positron energy, positron momentum (with $E=k^{2} / 2$ ), and positron-electron contact density, respectively. The positronelectron contact density was assumed to be independent of the nuclear coordinate and was calculated using the following equation:

$$
\rho_{\mathrm{ep}}=\int \gamma(r)\left|\psi_{i}(r)\right|^{2} \rho(r) d r
$$

where $\gamma(r)$ is an enhancement factor that can account for the short-range positron-electron Coulomb attraction. We employ the following expression proposed by Barbiellini et al. [41]:

$$
\begin{aligned}
\gamma(r)= & 1+\left(1.23 r_{\mathrm{s}}-0.0742 r_{\mathrm{s}}^{2}+\frac{1}{6} r_{\mathrm{s}}^{3}\right) \\
& \times \exp \left[-\beta|\nabla \rho|^{2} /\left(\rho q_{\mathrm{TF}}\right)^{2}\right],
\end{aligned}
$$

where $r_{\mathrm{s}}$ is the electron density parameter with $r_{\mathrm{s}}=$ $(3 / 4 \pi \rho)^{1 / 3}$.

The positron-molecule interaction of Eq. (2) can be conveniently calculated once the electron density and polarizability tensor are given. These quantities can be obtained from quantum chemistry calculations using the GAUSSIAN09 program package [52]. In this work we mainly employed DFT calculations with the $\omega$ B97XD [53] and Perdew-Burke-Ernzerhof (PBE) [54] exchange-correlation functionals. We found that these DFT functionals combined with the aug-cc-pVDZ basis set generally yield good agreement for the dipole moments and isotropic polarizabilities between theory and experiment. This agreement indirectly indicates the accuracy of 
TABLE I. Comparison of the calculated positron binding energy $\left(E_{\mathrm{b}}\right)$, dipole moment $(\mu)$, and isotropic polarizability $(\alpha)$ and corresponding experimental values.

\begin{tabular}{|c|c|c|c|c|}
\hline Molecule & Method $^{\mathrm{a}}$ & $\begin{array}{l}\text { Positron binding energy } \\
\qquad E_{\mathrm{b}} / \mathrm{meV}\end{array}$ & $\begin{array}{c}\text { Dipole moment } \\
\mu / \mathrm{D}\end{array}$ & $\begin{array}{c}\text { Polarizability } \\
\qquad \alpha / a_{0}^{3}\end{array}$ \\
\hline \multirow[t]{4}{*}{$\mathrm{C}_{2} \mathrm{H}_{3} \mathrm{Cl}$} & $\omega \mathrm{B} 97 \mathrm{XD}(\beta=0.21)$ & 28 & 1.591 & 40.73 \\
\hline & $\operatorname{PBE}(\beta=0.23)$ & 27 & 1.414 & 42.33 \\
\hline & $\operatorname{CCSD}(\beta=0.20)$ & 24 & 1.550 & 39.89 \\
\hline & Exp. & & $1.45^{\mathrm{b}}$ & $43.3^{\mathrm{b}}$ \\
\hline \multirow[t]{4}{*}{$1,1-\mathrm{C}_{2} \mathrm{H}_{2} \mathrm{Cl}_{2}$} & $\omega \mathrm{B} 97 \mathrm{XD}(\beta=0.21)$ & 24 & 1.478 & 53.66 \\
\hline & $\operatorname{PBE}(\beta=0.23)$ & 25 & 1.280 & 56.09 \\
\hline & $\operatorname{CCSD}(\beta=0.20)$ & 22 & 1.443 & 52.72 \\
\hline & Exp. & $35 \pm 3^{c}$ & $1.34^{\mathrm{b}}$ & $52.8^{\mathrm{b}}$ \\
\hline \multirow[t]{4}{*}{ cis- $\mathrm{C}_{2} \mathrm{H}_{2} \mathrm{Cl}_{2}$} & $\omega \mathrm{B} 97 \mathrm{XD}(\beta=0.21)$ & 84 & 2.020 & 53.35 \\
\hline & $\operatorname{PBE}(\beta=0.23)$ & 80 & 1.809 & 55.67 \\
\hline & $\operatorname{CCSD}(\beta=0.20)$ & 79 & 1.981 & 52.35 \\
\hline & Exp. & $76 \pm 1^{c}$ & $1.90^{\mathrm{b}}$ & $54.2^{\mathrm{b}}$ \\
\hline \multirow[t]{4}{*}{ trans $-\mathrm{C}_{2} \mathrm{H}_{2} \mathrm{Cl}_{2}$} & $\omega \mathrm{B} 97 \mathrm{XD}(\beta=0.21)$ & 8 & 0 & 54.79 \\
\hline & $\operatorname{PBE}(\beta=0.23)$ & 10 & 0 & 57.38 \\
\hline & $\operatorname{CCSD}(\beta=0.20)$ & 8 & 0 & 53.67 \\
\hline & Exp. & $15^{\mathrm{d}}(29 \pm 2)^{\mathrm{c}}$ & 0 & $55.0^{\mathrm{b}}$ \\
\hline \multirow[t]{4}{*}{$\mathrm{C}_{2} \mathrm{HCl}_{3}$} & $\omega \mathrm{B} 97 \mathrm{XD}(\beta=0.00)$ & 54 & 0.937 & 67.52 \\
\hline & $\operatorname{PBE}(\beta=0.03)$ & 64 & 0.822 & 70.90 \\
\hline & $\operatorname{CCSD}(\beta=0.00)$ & 36 & 0.920 & 66.25 \\
\hline & Exp. & $60 \pm 1^{c}$ & $0.8^{\mathrm{b}}$ & $65.8^{\mathrm{b}}$ \\
\hline \multirow[t]{4}{*}{$\mathrm{C}_{2} \mathrm{Cl}_{4}$} & $\omega \mathrm{B} 97 \mathrm{XD}(\beta=0.00)$ & 40 & 0 & 81.09 \\
\hline & $\operatorname{PBE}(\beta=0.03)$ & 54 & 0 & 85.34 \\
\hline & $\operatorname{CCSD}(\beta=0.00)$ & 26 & 0 & 79.54 \\
\hline & Exp. & $57^{d}(65 \pm 2)^{c}$ & 0 & $81.1^{\mathrm{e}}$ \\
\hline
\end{tabular}

${ }^{\text {a }}$ The aug-cc-pVDZ basis set was used for all three electronic structure calculations.

${ }^{b}$ Experimental data taken from Ref. [63].

${ }^{\mathrm{c}}$ Taken from Ref. [44], where annihilation spectra with 40-50 meV resolution were reported.

${ }^{\mathrm{d}}$ Taken from Ref. [13], where annihilation spectra with $20 \mathrm{meV}$ resolution were reported.

${ }^{\text {e}}$ Experimental data (in solution) taken from Ref. [64].

the electron densities calculated with these DFT functionals. Nevertheless, in order to further confirm the accuracy of these DFT functionals, we additionally calculated the positron binding energies using the highly correlated coupled cluster with single and double excitation (CCSD) method with the same aug-cc-pVDZ basis set. Recent benchmark studies have indicated that the CCSD method provides very accurate electron densities, dipole moments, and dipole polarizabilities for many closed-shell molecules [55-58].

The Schrödinger equation for the positron motion was solved using the time-dependent wave-packet formalism for both the bound and scattering states, where the wave packet was described using the Cartesian discrete variable representation (DVR) grid method [59] with the particle-in-a-box basis functions. We have solved the time-dependent Schrödinger equations using the well-known fast-Fourier transform algorithm combined with the split-operator method [60,61]. As for the bound-state problem, we have applied the direct relaxation method [62], where the initial trial wave packet is propagated in imaginary time, which finally leads to the bound positron state. We applied a cut-off energy parameter of 20 hartree to the total positron-molecule potential to avoid divergent Coulomb repulsion from positive nuclei. We employed $(256)^{3}$ grid points in the range of $-L / 2 \leqslant(x, y, z) \leqslant L / 2$, where the center of mass of the target molecule was taken as the coordinate origin and the box size $L$ was varied in the range of 40-100 $a_{0}$ to obtain fully converged results. Details of our computational procedures were also described in Refs. [38-40].

\section{RESULTS AND DISCUSSION}

Before presenting the calculated annihilation spectra, we discuss the determination of the $\beta$ value [Eq. (4)] in the generalized gradient approximation on the basis of the measured positron binding energies. As mentioned previously, $\beta$ was assumed to be an adjustable parameter and can thus be optimized such that the calculated positron binding energy matches the measured positron binding energy for each molecule. However, it would be desirable to employ a uniform $\beta$ value to calculate the positron binding energies for the different molecules. For this purpose we first 


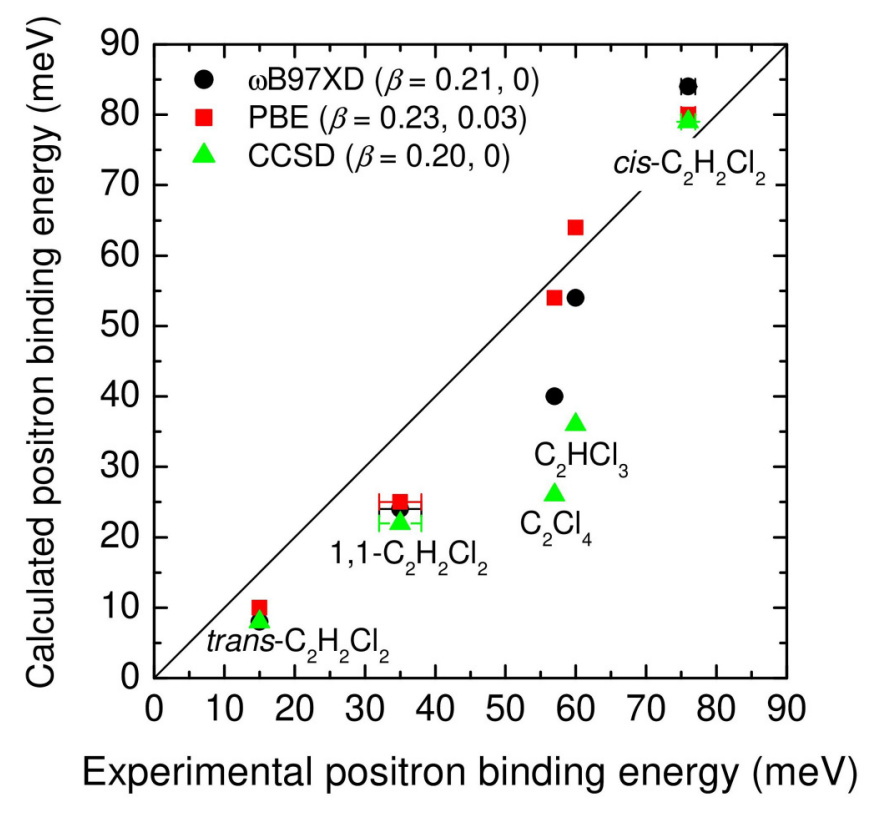

FIG. 1. Comparison of calculated positron binding energies and experimental data. The generalized gradient approximation parameter $(\beta)$ was optimized for each electronic structure level $(\omega \mathrm{B} 97 \mathrm{XD}$, PBE, or CCSD) (see the text for details).

determined the $\beta$ value using the experimental values for the three dichloroethene isomers, namely, 1,1-dichloroethene, cis-dichloroethene, and trans-dichloroethene. This was considered as a reasonable choice, because these three molecules have the same stoichiometry. The value of $\beta$ was determined using each density functional or electronic structure method ( $\omega$ B97XD, PBE, or CCSD) such that the root-mean-square deviation was minimized. The results are summarized in Table I along with the molecular properties including dipole moments and isotropic polarizabilities taken from Refs. [63,64]. Figure 1 presents a comparison of the calculated positron binding energies and the experimental values. We found that the positron binding energies calculated using the $\omega \mathrm{B} 97 \mathrm{XD}, \mathrm{PBE}$, and CCSD methods reasonably reproduced the measured values for the three dichloroethenes. The $\beta$ values were optimized to be $0.21,0.23$, and 0.20 for the $\omega \mathrm{B} 97 \mathrm{XD}, \mathrm{PBE}$, and CCSD methods, respectively. It is interesting to note that these $\beta$ values are close to the value proposed in Ref. [41] $(\beta=0.22)$. Among the three electronic structure methods, it can be seen that the PBE method afforded excellent agreement.

The positron binding energy was largest for cisdichloroethene and smallest for trans-dichloroethene. Thus it can be seen that the positron binding energy was positively correlated with the magnitude of the dipole moment. In order to further elucidate the positron binding mechanism, we present the calculated positron densities and positronmolecule interaction potentials in Fig. 2, where we have shown only the PBE results. In the case of cis-dichloroethene, the most attractive potential well was observed around the negative end of the dipole moment vector, which is close to the two chlorine atoms. Another interesting feature is that

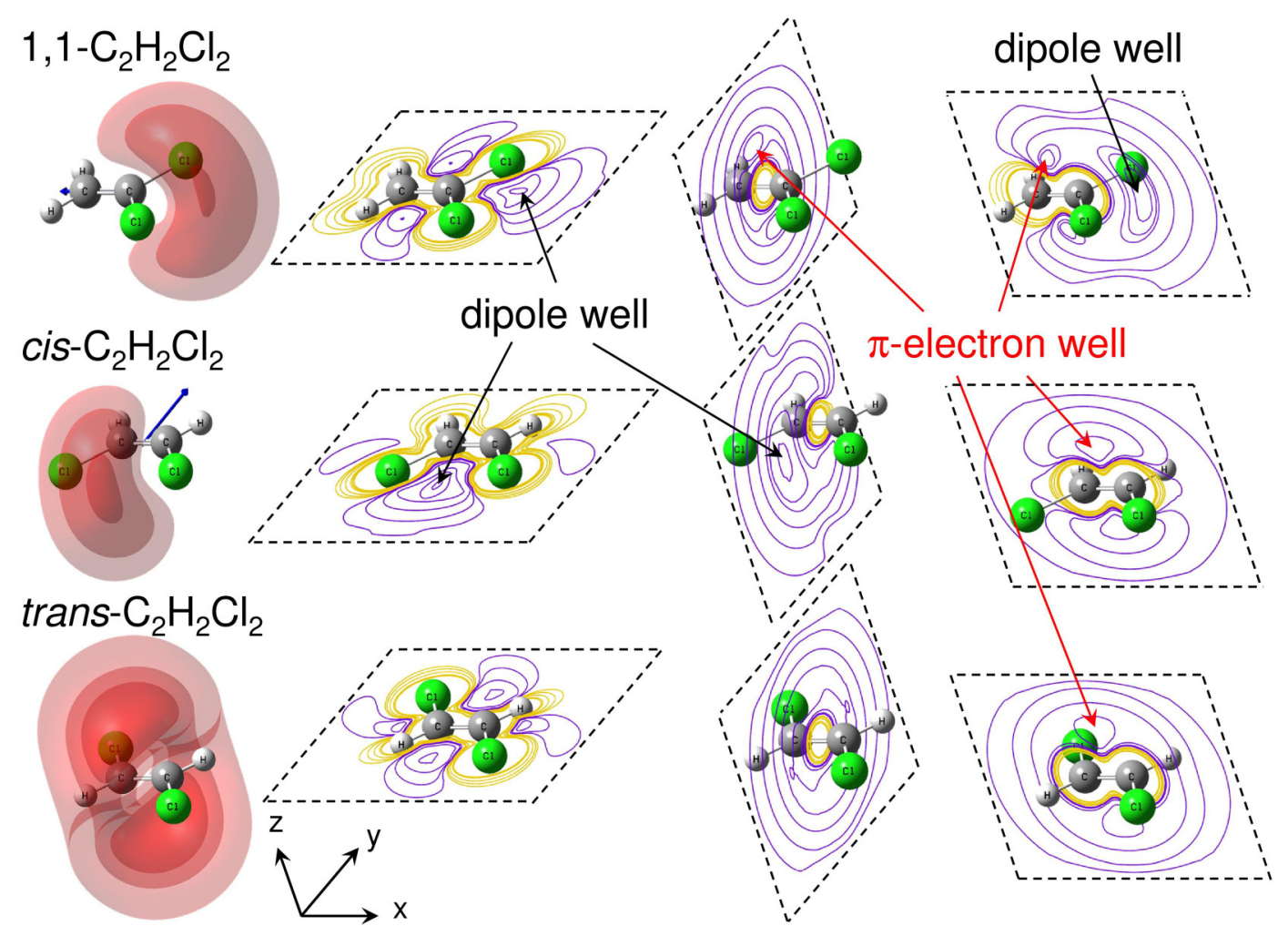

FIG. 2. Positron densities (left panels) and positron-molecule interaction potentials projected on the three planes $(x, y, z=0),(x, y=0, z)$, and $(x=0, y, z)$ for 1,1-dichloroethene, cis-dichloroethene, and trans-dichloroethene, respectively. The calculations were performed using the PBE functional with the aug-cc-pVDZ basis set. Positive (repulsive) and negative (attractive) energy contours are indicated with yellow (light gray) and purple (dark gray) contour lines, respectively. The potential energy wells due to the dipole moment and $\pi$ electrons are also indicated. 


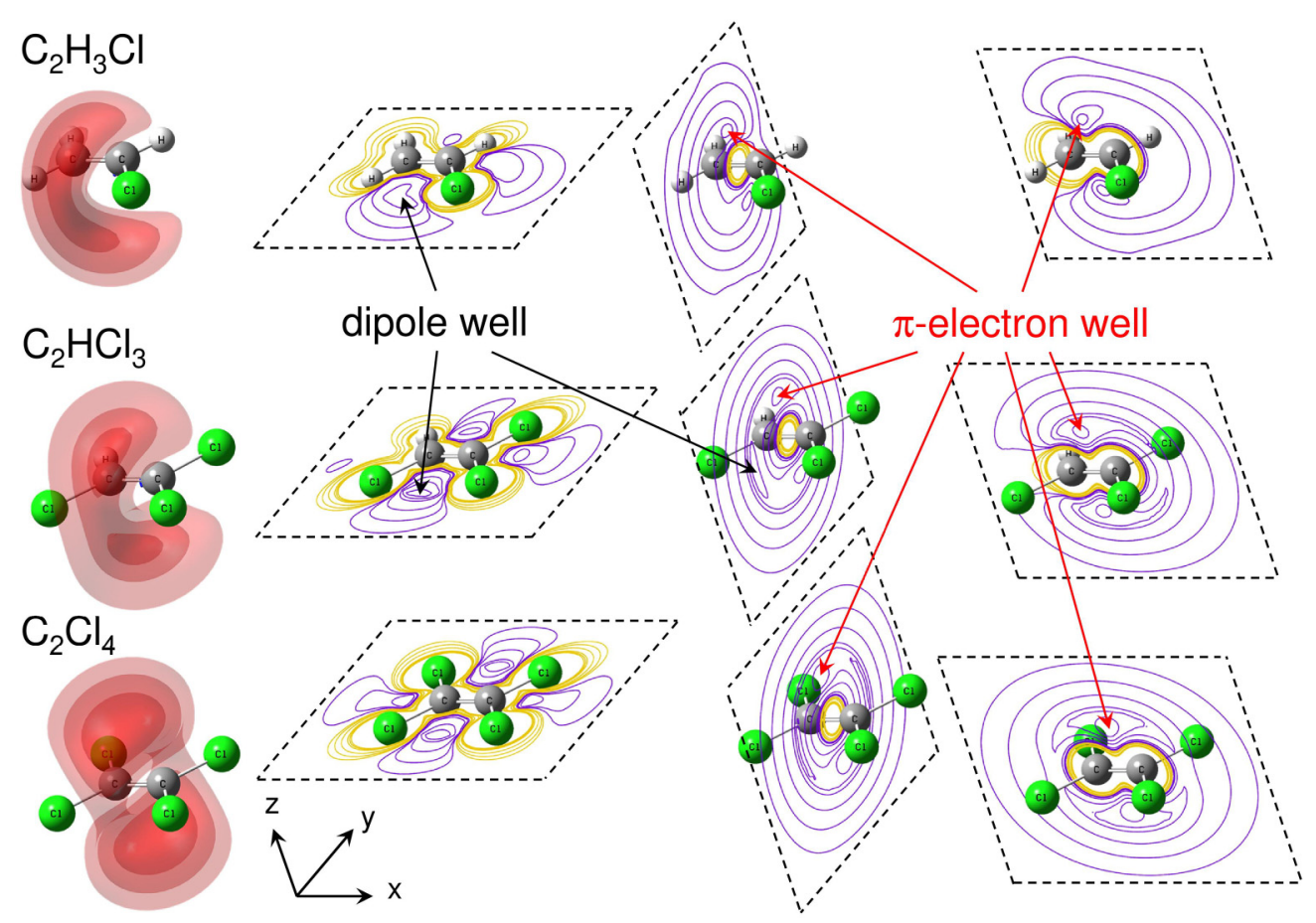

FIG. 3. Similar plots to Fig. 2 for vinyl chloride, trichloroethene, and tetrachloroethene.

potential energy wells can also be observed around the areas close to the $\mathrm{C}=\mathrm{C} \pi$ bond. Hence we can see that the positron density was relatively high around these potential energy wells. This indicates that positron binding is primarily determined by the attractive dipole field and that $\pi$ electrons enhance the binding. A similar trend can be observed for 1,1-dichloroethene, although the dipole attraction was somewhat weaker than that for cis-dichloroethene. Again, it can be seen that the positron was attracted by the $\pi$ electrons. In addition to the combination of the dipole field and attractive interaction by $\pi$ electrons, the importance of $\pi$ electrons in positron binding can be most clearly understood for nonpolar trans-dichloroethene, which has zero dipole moment due to symmetry. In this case the positron was found to be exclusively bound around the $\mathrm{C}=\mathrm{C} \pi$ bond. These results thus demonstrate the importance of $\pi$ electrons in positron binding.

As mentioned previously, it would be preferable to use the same $\beta$ parameter for different molecules such that the CPP model is universal. However, the calculated binding energies for trichloroethene and tetrachloroethene were found to be significantly underestimated compared to the experimental data when we employed the $\beta$ value determined from the experimental results for the three dichloroethenes. We therefore decided to use a newly optimized $\beta$ value for trichloroethene and tetrachloroethene. The obtained results are also presented in Table I and Fig. 1. In this case, the optimized $\beta$ values for the three electronic structure methods $(\omega \mathrm{B} 97 \mathrm{XD}, \mathrm{PBE}$, and CCSD) were found to be in the range of $0-0.05$, which is in good agreement with the value used in Ref. [42] $(\beta=0.05)$. The difference of $\beta$ value between dichloroethenes and larger chloroethenes (trichloroethene and tetrachloroethene) suggests that one should employ different $\beta$ values depending on the dipole polarizability. In fact, from the results presented in Table I, the polarizability values for trichloroethene and tetrachloroethene are larger than those for other chloroethenes. However, the correlation between the optimal value of $\beta$ in positron binding and the molecular properties should be studied more systematically for various molecules in the near future.

From the results present in Table I, an appropriate choice of $\beta$ may be correlated with the dipole polarizability of the molecule, although further theoretical studies should be required to confirm this.

The calculated positron densities for other three chloroethene molecules are shown in Fig. 3 along with the positron-molecule interaction potentials. Again, one can see the attractive potential wells due to $\pi$ electrons for the three molecules. It is interesting to notice that, in the case of the nonpolar tetrachloroethene case, the positron is bound only around the attractive wells due to the $\pi$ electron. This feature may be highly correlated with the importance of the $\mathrm{C}=\mathrm{C}$ stretch mode in the annihilation spectrum, as will be shown below.

We next compare the calculated annihilation spectra to the experimental spectra. Here we present only the results for trans-dichloroethene and tetrachloroethene, because highresolution annihilation spectra are available for these two nonpolar molecules. We have calculated the annihilation spectra for all six chloroethene molecules, and the calculated spectra for the other molecules are provided in the Supplemental Material [65]. In addition, we here present the annihilation spectra calculated using the PBE functionals. The results calculated using the $\omega \mathrm{B} 97 \mathrm{XD}$ and CCSD methods are provided in the Supplemental Material [65], although the CCSD result is presented only for vinylchloride due to the computational cost. We found that the vibrational frequencies (especially for high-frequency modes) calculated 


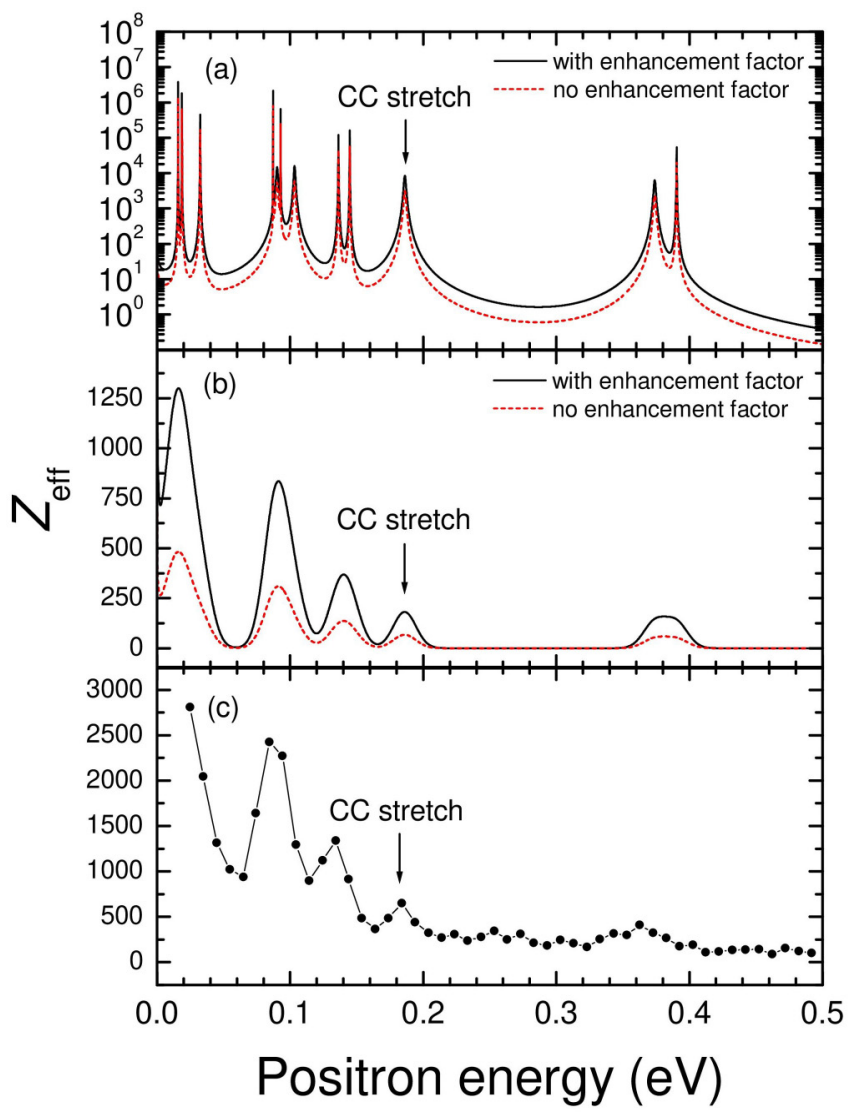

FIG. 4. Comparison of the experimental and theoretical annihilation spectra of trans-dichloroethene. Panel (a) shows the calculated spectra without convolution. Panel (b) shows the calculated spectra convoluted with a Gaussian function of $20 \mathrm{meV}$ width. The solid black and dotted red lines indicate the results with and without an enhancement factor, respectively. The calculations were performed using the PBE functional with the aug-cc-pVDZ basis set. Panel (c) shows the experimental spectrum taken from Ref. [13]. with the $\omega$ B97XD functional for neutral molecules are somewhat overestimated compared to the experimental values. Notice that the accuracy of the vibrational frequencies is an important point, since the annihilation resonance positions significantly depend on the vibrational energy levels of the neutral molecule. This suggests that the choice of an appropriate DFT functional is important for obtaining good agreement between theory and experiment.

Figure 4 shows a comparison between theory and experiment for trans-dichloroethene. The calculated resonance widths are summarized in Table II along with the vibrational mode assignment taken from Ref. [66]. The calculated spectrum without energy convolution for trans-dichloroethene is shown in Fig. 4(a). The calculated spectrum convoluted with a Gaussian distribution of $20 \mathrm{meV}$ width is shown in Fig. 4(b), which can be compared to the high-resolution experimental spectrum presented in Fig. 4(c). Good agreement can be seen between theory and experiment except for the absolute values. Notice that we here show the calculated spectrum with and without an enhancement factor [41,67] to confirm the effect of this factor on the spectral intensity The introduction of the enhancement factor significantly increased the spectral intensity by a factor of about 3 ; however, the calculated peak intensity with the enhancement factor was found to be still smaller than the experimental ones by a factor of about 3 . This indicates that the absolute value of the annihilation rate should be carefully determined using a more accurate enhancement factor. In addition to this improvement, it is worth mentioning that intramolecular vibrational redistribution [5,12], which was neglected in this work, should be taken into account to evaluate the magnitude of the annihilation intensities at a more quantitative level.

The most important finding is that the calculated spectrum displayed a clear peak at $E=186 \mathrm{meV}$, which can be favorably compared to the experimental peak at $E \sim 185 \mathrm{meV}$. This peak is associated with the infrared-inactive $\mathrm{C}=\mathrm{C}$ stretch (see Table II). A previous experimental study [13] has

TABLE II. Resonance energies and widths of trans-dichloroethene for each vibrational state calculated using the CPP model including the generalized gradient approximation. The characters of each vibrational mode are also indicated.

\begin{tabular}{|c|c|c|c|c|}
\hline $\begin{array}{l}\text { Mode }^{\mathrm{a}} \\
\text { (Sym.) }\end{array}$ & $\begin{array}{l}\text { Resonance energy } \\
\text { (Energy shift) }^{\mathrm{b}} / \mathrm{eV}\end{array}$ & $\begin{array}{l}\text { Resonance width } \\
\qquad / \mathrm{au}^{\mathrm{c}}\end{array}$ & $\begin{array}{c}\text { Infrared/Raman } \\
\text { Activity }\end{array}$ & Vibration type \\
\hline$v_{7}\left(a_{\mathrm{u}}\right)$ & $0.016(0.010)$ & $2.97(-7)$ & Active / Inactive & Torsion \\
\hline$v_{12}\left(b_{\mathrm{u}}\right)$ & $0.019(0.010)$ & $5.10(-7)$ & Active / Inactive & $\mathrm{CCCl}$ deformation \\
\hline$v_{5}\left(a_{\mathrm{g}}\right)$ & $0.032(0.010)$ & $1.04(-6)$ & Inactive / Active & $\mathrm{CCCl}$ deformation \\
\hline$v_{8}\left(b_{\mathrm{g}}\right)$ & $0.087(0.010)$ & $5.85(-8)$ & Inactive / Active & $\mathrm{CH}$ bend \\
\hline$v_{11}\left(b_{\mathrm{u}}\right)$ & $0.090(0.010)$ & $8.19(-6)$ & Active / Inactive & $\mathrm{CCl}$ stretch \\
\hline$v_{4}\left(a_{\mathrm{g}}\right)$ & $0.093(0.010)$ & $1.77(-7)$ & Inactive / Active & $\mathrm{CCl}$ stretch \\
\hline$v_{6}\left(a_{\mathrm{u}}\right)$ & $0.103(0.010)$ & $6.33(-6)$ & Active / Inactive & $\mathrm{CH}$ bend \\
\hline$v_{10}\left(b_{\mathrm{u}}\right)$ & $0.136(0.010)$ & $5.59(-7)$ & Active / Inactive & $\mathrm{CH}$ bend \\
\hline$v_{3}\left(a_{\mathrm{g}}\right)$ & $0.145(0.010)$ & $3.84(-7)$ & Inactive / Active & $\mathrm{CH}$ bend \\
\hline$v_{2}\left(a_{\mathrm{g}}\right)$ & $0.186(0.010)$ & $5.04(-6)$ & Inactive / Active & $\mathrm{CC}$ stretch \\
\hline$v_{1}\left(a_{\mathrm{g}}\right)$ & $0.374(0.010)$ & $2.42(-6)$ & Inactive / Active & $\mathrm{CH}$ stretch \\
\hline$v_{9}\left(b_{\mathrm{u}}\right)$ & $0.390(0.010)$ & $2.71(-7)$ & Active / Inactive & $\mathrm{CH}$ stretch \\
\hline
\end{tabular}

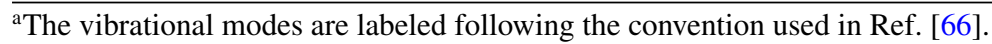

${ }^{b}$ Energy shift measured from the neutral vibrational state with the same quantum number.

${ }^{\mathrm{c}}$ The notation $a(b)$ denotes $a \times 10^{b}$. 


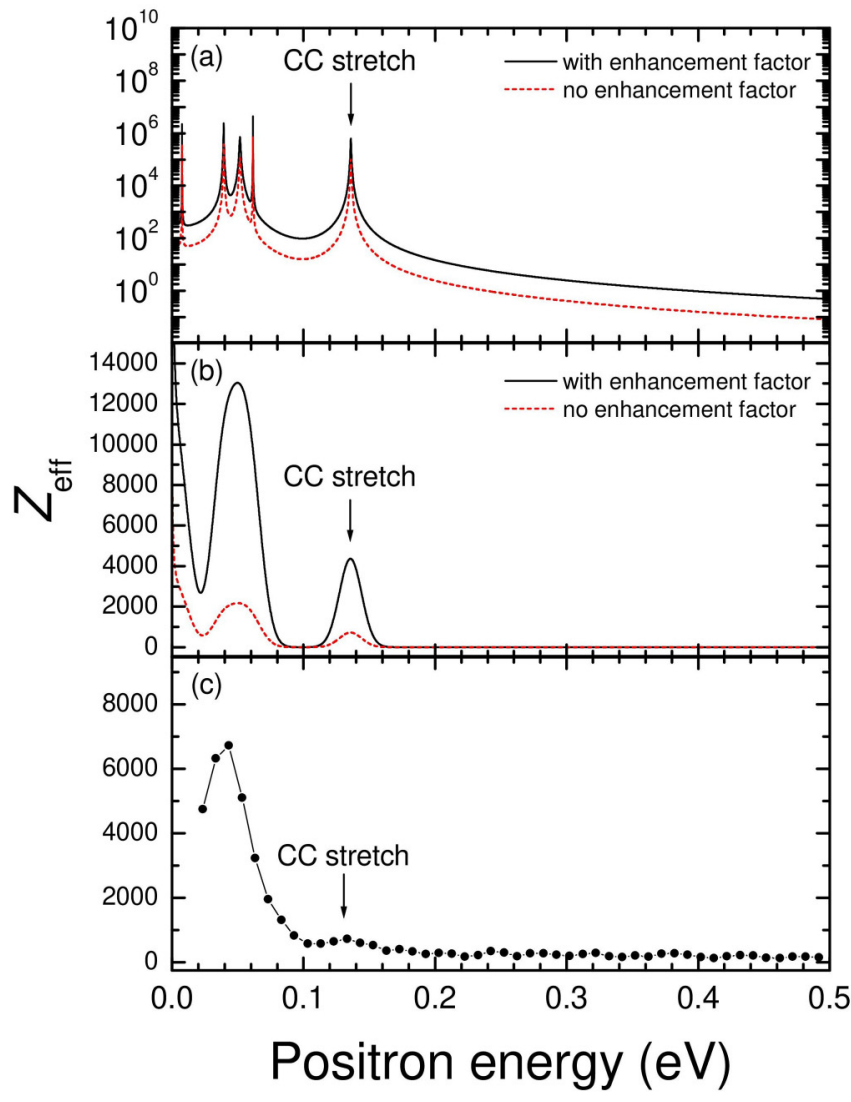

FIG. 5. Similar plots to Fig. 4 for tetrachloroethene.

already demonstrated that this peak cannot be reproduced by Gribakin-Lee theory taking only the infrared-active (dipoleallowed) transitions into account [50]. It is worth mentioning that the current CPP model can describe the contribution of the infrared-inactive vibrations through the potential coupling term [see Eq. (6)]. One important point is that the infraredinactive $\mathrm{C}=\mathrm{C}$ stretch exhibited the greatest width among the infrared-inactive modes as listed in Table II. This behavior can be qualitatively understood from the fact that the positron is predominantly bound by $\mathrm{C}=\mathrm{C} \pi$ electrons in the nonpolar trans-dichloroethene molecule (see Fig. 2). In addition to this, the $\mathrm{C}=\mathrm{C}$ stretching mode is Raman active, but this mode did not afford the strongest transition in the measured Raman spectrum; the strongest transition was associated with the symmetric $\mathrm{C}-\mathrm{H}$ stretch [68]. The present finding thus suggests that the $\mathrm{C}=\mathrm{C} \pi$ bond plays a more essential role in positron binding than the overall polarizability change through vibrational motion.

The importance of the $\pi$ bond can also be observed in the results for tetrachloroethene. Figure 5 presents a comparison of the calculated annihilation spectrum and a high-resolution experimental spectrum. The calculated resonance widths are summarized in Table III. Similar to the case of trans-dichloroethene in Fig. 4, it was found that the enhancement factor increased the spectral intensity by a factor of about 6 . In this case, however, the calculated intensity with the enhancement factor is somewhat higher than the experimental intensity. Again, a more accurate treatment for the enhancement factor will be required. It is worth mentioning that Swann and Gribakin have recently employed a different functional form to estimate the enhancement factor [69]. A broad peak in the experimental spectrum at $E \sim 135 \mathrm{meV}$, which corresponds to the infrared-inactive $\mathrm{C}=\mathrm{C}$ stretch, was reasonably reproduced in the calculated spectra. Again, it is worth mentioning that this peak cannot be reproduced by the Gribakin-Lee model [50]. Therefore, the present CPP model can describe the contribution of this dipole-forbidden transition to the annihilation spectrum, and we conclude that the importance of the $\mathrm{C}=\mathrm{C}$ stretch in the annihilation spectrum is correlated with the role of $\pi$ electrons in positron binding.

\section{CONCLUSIONS}

In summary, we have theoretically investigated the applicability of the practical CPP model for describing the positron binding phenomenon in a series of six chloroethene molecules. The experimentally determined positron binding energies were reasonably reproduced by the CPP model when we employed an appropriate $\beta$ value, which controls the contribution of the gradient correction to the short-range positronelectron potential. From detailed analyses of the positron densities and positron-electron interaction potentials, we found that $\pi$ electrons play an important role in positron binding, although the dominant interaction is the positron-dipole interaction. The importance of $\pi$ electrons has also been demonstrated through comparison of the calculated positronelectron annihilation spectra and the experimental spectra for nonpolar trans-dichloroethene and tetrachloroethene. The dominant resonance peaks corresponded to infrared-active

TABLE III. Resonance energies and widths of tetrachloroethene for each vibrational state calculated using the CPP model including the generalized gradient approximation. The characters of each vibrational mode are also indicated.

\begin{tabular}{|c|c|c|c|c|}
\hline $\begin{array}{l}\text { Mode }^{\mathrm{a}} \\
\text { (Sym.) }\end{array}$ & $\begin{array}{l}\text { Resonance energy } \\
(\text { Energy shift })^{\mathrm{b}} / \mathrm{eV}\end{array}$ & $\begin{array}{l}\text { Resonance width } \\
\qquad / \mathrm{au}^{\mathrm{c}}\end{array}$ & $\begin{array}{c}\text { Infrared/Raman } \\
\text { Activity }\end{array}$ & Vibration type \\
\hline$v_{8}\left(b_{2 \mathrm{~g}}\right)$ & $0.008(0.054)$ & $2.10(-8)$ & inactive / active & $\mathrm{CCl}_{2}$ wagging \\
\hline$v_{11}\left(b_{3 \mathrm{u}}\right)$ & $0.039(0.054)$ & $1.03(-5)$ & active / inactive & $\mathrm{CCl}_{2}$ stretch \\
\hline$v_{9}\left(b_{2 u}\right)$ & $0.052(0.055)$ & $3.12(-5)$ & active / inactive & $\mathrm{CCl}_{2}$ stretch \\
\hline$v_{5}\left(b_{1 \mathrm{~g}}\right)$ & $0.062(0.054)$ & $5.28(-7)$ & inactive / active & $\mathrm{CCl}_{2}$ stretch \\
\hline$v_{1}\left(a_{\mathrm{g}}\right)$ & $0.136(0.055)$ & $2.16(-5)$ & inactive / active & $\mathrm{CC}$ stretch \\
\hline
\end{tabular}

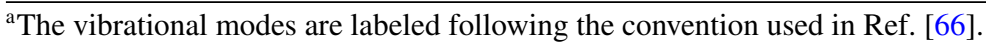

${ }^{\mathrm{b}}$ Energy shift measured from the neutral vibrational state with the same quantum number.

${ }^{\mathrm{c}}$ The notation $a(b)$ denotes $a \times 10^{b}$.
} 
(dipole-allowed) vibrational motions; however, the infraredinactive $\mathrm{C}=\mathrm{C}$ stretching mode also contributed to the annihilation spectra. This result is in accordance with experimental findings [13] and suggests the importance of $\pi$ electrons in positron binding in polyatomic molecules.

\section{ACKNOWLEDGMENT}

This work was supported by a Grant-in-Aid for Scientific Research from the Ministry of Education, Culture, Sports, Science and Technology of Japan (Grant No. 19K05365).
[1] G. F. Gribakin, J. A. Young, and C. M. Surko, Rev. Mod. Phys. 82, 2557 (2010).

[2] L. D. Barnes, S. J. Gilbert, and C. M. Surko, Phys. Rev. A 67, 032706 (2003).

[3] L. D. Barnes, J. A. Young, and C. M. Surko, Phys. Rev. A 74, 012706 (2006).

[4] J. A. Young and C. M. Surko, Phys. Rev. Lett. 99, 133201 (2007).

[5] J. A. Young and C. M. Surko, Phys. Rev. A 77, 052704 (2008).

[6] J. A. Young and C. M. Surko, Phys. Rev. A 78, 032702 (2008).

[7] J. A. Young and C. M. Surko, Phys. Status Solidi C 6, 2265 (2009).

[8] J. R. Danielson, J. A. Young, and C. M. Surko, J. Phys. B: At. Mol. Opt. Phys. 42, 235203 (2009).

[9] J. R. Danielson, J. J. Gosselin, and C. M. Surko, Phys. Rev. Lett. 104, 233201 (2010).

[10] J. R. Danielson, A. C. L. Jones, M. R. Natisin, and C. M. Surko, Phys. Rev. Lett. 109, 113201 (2012).

[11] J. R. Danielson, A. C. L. Jones, J. J. Gosselin, M. R. Natisin, and C. M. Surko, Phys. Rev. A 85, 039907(E) (2012).

[12] A. C. L. Jones, J. R. Danielson, J. J. Gosselin, M. R. Natisin, and C. M. Surko, New J. Phys. 14, 015006 (2012).

[13] M. R. Natisin, J. R. Danielson, G. F. Gribakin, A. R. Swann, and C. M. Surko, Phys. Rev. Lett. 119, 113402 (2017).

[14] S. L. Saito and F. Sasaki, J. Chem. Phys. 102, 8040 (1995).

[15] M. Tachikawa, Chem. Phys. Lett. 350, 269 (2001).

[16] M. W. J. Bromley and J. Mitroy, Phys. Rev. A 65, 062505 (2002).

[17] K. Strasburger, J. Chem. Phys. 114, 615 (2001).

[18] M. Mella and G. Morosi, J. Chem. Phys. 113, 6154 (2000).

[19] J. Mitroy, Phys. Rev. A 73, 054502 (2006).

[20] D. M. Schrader, T. Yoshida, and K. Iguchi, Phys. Rev. Lett. 68, 3281 (1992).

[21] N. Jiang and D. M. Schrader, J. Chem. Phys. 109, 9430 (1998).

[22] D. Bressanini, M. Mella, and G. Morosi, J. Chem. Phys. 108, 4756 (1998).

[23] M. Mella, M. Casalegno, and G. Morosi, J. Chem. Phys. 117, 1450 (2002).

[24] F. A. Gianturco, T. Mukherjee, and P. Paioletti, Phys. Rev. A 56, 3638 (1997).

[25] F. A. Gianturco, T. Mukherjee, and A. Occhigrossi, Phys. Rev. A 64, 032715 (2001).

[26] T. Nishimura and F. A. Gianturco, Phys. Rev. A 65, 062703 (2002).

[27] T. Nishimura and F. A. Gianturco, Phys. Rev. Lett. 90, 183201 (2003).

[28] F. A. Gianturco, T. L. Gibson, P. Nichols, R. R. Lucchese, and T. Nishimura, Rad. Phys. Chem. 68, 673 (2003).

[29] T. Nishimura and F. A. Gianturco, J. Phys. B: At. Mol. Opt. Phys. 37, 215 (2004).
[30] T. Nishimura and F. A. Gianturco, Eur. Phys. J. D 33, 221 (2005).

[31] T. Nishimura and F. A. Gianturco, Phys. Rev. A 72, 022706 (2005).

[32] F. A. Gianturco, P. Nichols, T. L. Gibson, and R. R. Lucchese, Phys. Rev. A 72, 032724 (2005).

[33] J. Franz and F. A. Gianturco, Eur. Phys. J. D 68, 279 (2014).

[34] F. Carelli, F. A. Gianturco, J. Franz, and M. Satta, Eur. Phys. J. D 69, 143 (2015).

[35] K. Fedus, J. Franz, and G. P. Karwasz, Phys. Rev. A 91, 062701 (2015).

[36] F. A. Gianturco and R. R. Lucchese, Phys. Rev. A 60, 4567 (1999).

[37] R. Carey, R. R. Lucchese, and F. A. Gianturco, Phys. Rev. A 78, 012706 (2008).

[38] Y. Sugiura, T. Takayanagi, Y. Kita, and M. Tachikawa, Eur. Phys. J. D 73, 162 (2019).

[39] Y. Sugiura, H. Suzuki, T. Otomo, T. Miyazaki, T. Takayanagi, and M. Tachikawa, J. Comp. Chem. 41, 1576 (2020).

[40] Y. Sugiura, T. Takayanagi, and M. Tachikawa, Int. J. Quant. Chem. 120, e26376 (2020).

[41] B. Barbiellini, M. J. Puska, T. Korhonen, A. Harju, T. Torsti, and R. M. Nieminen, Phys. Rev. B 53, 16201 (1996).

[42] J. Kuriplach and B. Barbiellini, Phys. Rev. B 89, 155111 (2014).

[43] G. F. Gribakin, J. F. Stanton, J. R. Danielson, M. R. Natisin, and C. M. Surko, Phys. Rev. A 96, 062709 (2017).

[44] M. R. Natisin, Ph.D. thesis, University of California, San Diego, 2016.

[45] E. Boroński and R. M. Nieminen, Phys. Rev. B 34, 3820 (1986).

[46] P. Villarreal, S. Miret-Artés, O. Roncero, G. Delgado-Barrio, J. A. Beswick, N. Halberstadt, and R. D. Coalson, J. Chem. Phys. 94, 4230 (1991).

[47] O. Roncero, N. Halberstadt, and J. A. Beswick, J. Chem. Phys. 104, 7554 (1996).

[48] D. H. Zhang and J. Z. H. Zhang, J. Chem. Phys. 95, 6449 (1991).

[49] D. H. Zhang, J. Z. H. Zhang, and Z. Bačić, J. Chem. Phys. 97, 927 (1992).

[50] G. F. Gribakin and C. M. R. Lee, Phys. Rev. Lett. 97, 193201 (2006).

[51] G. F. Gribakin and C. M. R. Lee, Eur. Phys. J. D 51, 51 (2009).

[52] M. J. Frisch, G. W. Trucks, H. B. Schlegel, G. E. Scuseria, M. A. Robb, J. R. Cheeseman, G. Scalmani, V. Barone, B. Mennucci, G. A. Petersson et al., Gaussian 09, Revision D.01, Gaussian, Inc., Wallingford, CT, 2009.

[53] J.-D. Chai and M. Head-Gordon, Phys. Chem. Chem. Phys. 10, 6615 (2008).

[54] J. P. Perdew, K. Burke, and M. Ernzerhof, Phys. Rev. Lett. 77, 3865 (1996). 
[55] A. L. Hickey and C. N. Rowley, J. Phys. Chem. A 118, 3678 (2014).

[56] T. Wu, Y. N. Kalugina, and A. J. Thakkar, Chem. Phys. Lett. 635, 257 (2015).

[57] D. Hait and M. Head-Gordon, J. Chem. Theo. Comp. 14, 1969 (2018).

[58] D. Hait and M. Head-Gordon, Phys. Chem. Chem. Phys. 20, 19800 (2018).

[59] J. C. Light, I. P. Hamilton, and J. V. Lill, J. Chem. Phys. 82, 1400 (1985).

[60] M. D. Feit, J. A. Fleck, Jr., and A. Steiger, J. Comp. Phys. 47, 412 (1982).

[61] G. Nyman, Int. J. Quantum Chem. 114, 1183 (2014).

[62] R. Kosloff and H. Tal-Ezer, Chem. Phys. Lett. 127, 223 (1986).

[63] National Institute of Standards and Technology, Computational Chemistry Comparison and Benchmark DataBase, Release
20 (August 2019), Standard Reference Database 101, https: //cccbdb.nist.gov/ (accessed on Aug. 5, 2020).

[64] CRC Handbook of Chemistry and Physics, 94th ed., edited by W. M. Haynes (CRC Press, Boca Raton, FL, 2013).

[65] See Supplemental Material at http://link.aps.org/supplemental/ 10.1103/PhysRevA.102.052830 for other results calculated using different DFT functionals.

[66] NIST Chemistry Webbook, https://webbook. nist.gov/chemistry/ (accessed on Sep. 8, 2020).

[67] A. R. Swann and G. F. Gribakin, J. Chem. Phys. 149, 244305 (2018).

[68] B. L. McClain, S. M. Clark, R. L. Gabriel, and D. Ben-Amotz, J. Chem. Educ. 77, 654 (2000).

[69] A. R. Swann and G. F. Gribakin, Phys. Rev. A 101, 022702 (2020). 